\title{
Diffusion-Weighted Imaging of Nasopharyngeal Carcinoma: Can Pretreatment DWI Predict Local Failure Based on Long-Term Outcome?
}

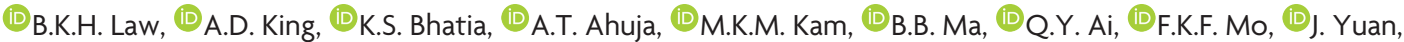 \\ and $\oplus^{D}$ D.K.W. Yeung
}

\begin{abstract}
BACKGROUND AND PURPOSE: Pretreatment prediction of patients with nasopharyngeal carcinoma who will fail conventional treatment would potentially allow these patients to undergo more intensive treatment or closer posttreatment monitoring. The aim of the study was to determine the ability of pretreatment DWI to predict local failure in patients with nasopharyngeal carcinoma based on long-term clinical outcome.
\end{abstract}

MATERIALS AND METHODS: One hundred fifty-eight patients with pretreatment DWI underwent analysis of the primary tumor to obtain the ADC mean, ADC skewness, ADC kurtosis, volume, and T-stage. Univariate and multivariate analyses using logistic regression were performed to compare the ADC parameters, volume, T-stage, and patient age in primary tumors with local failure and those with local control, by using a minimum of 5-year follow-up to confirm local control.

RESULTS: Local control was achieved in 131/158 (83\%) patients (range, 60.3-117.7 months) and local failure occurred in 27/158 (17\%) patients (range, 5.2-79.8 months). Compared with tumors with local control, those with local failure showed a significantly lower ADC skewness ( $A D C$ values with the greatest frequencies were shifted away from the lower $A D C$ range) $(P=.006)$ and lower ADC kurtosis (curve peak broader) $(P=.024)$. The ADC skewness remained significant on multivariate analysis $(P=.044)$. There was a trend toward higher tumor volumes in local failure, but the volume, together with T-stage and ADC mean, were not significantly different between the 2 groups.

CONCLUSIONS: Pretreatment DWI of primary tumors found that the skewness of the ADC distribution curve was a predictor of local failure in patients with nasopharyngeal carcinoma, based on long-term clinical outcome.

ABBREVIATIONS: $L C=$ local control; $L F=$ local failure; NPC = nasopharyngeal carcinoma; $R O C=$ receiver operating characteristic

$\mathbf{N}$ asopharyngeal carcinoma (NPC) is a radiosensitive tumor, but despite recent advances in treatment by using intensitymodulated radiation therapy, local tumor recurrence still occurs in $12 \%$ of patients. ${ }^{1}$ Recurrent primary tumors deep to the naso-

Received November 30, 2015; accepted after revision February 27, 2016.

From the Departments of Imaging and Interventional Radiology (B.K.H.L., A.D.K., K.S.B., A.T.A., Q.Y.A.) and Clinical Oncology (M.K.M.K., B.B.M., F.K.F.M., D.K.W.Y.), The Chinese University of Hong Kong, Prince of Wales Hospital, Hong Kong S.A.R., China; and Medical Physics and Research Department (J.Y.), Hong Kong Sanatorium and Hospital, Happy Valley, Hong Kong S.A.R., China.

We report the following author contributions: conception and design: B.K.H.L., A.D.K., D.K.W.Y.; acquisition of data: K.S.B., A.T.A., M.K.M.K., B.B.M., Q.Y.A., D.K.W.Y.; analysis and interpretation of data: B.K.H.L., A.D.K., F.K.F.M., J.Y.; drafting the article or revising it critically for important intellectual content: all authors; final approval of the version to be published: all authors; and agreement to be accountable for all aspects of the work in ensuring that questions related to the accuracy or integrity of any part of the work are appropriately investigated and resolved: all authors.

Please address correspondence to Ann D. King, FRCR, Department of Imaging and Interventional Radiology, Faculty of Medicine, The Chinese University of Hong Kong, Prince of Wales Hospital, 30-32 Ngan Shing St, Shatin, New Territories, Hong Kong S.A.R., China; e-mail: king2015@cuhk.edu.hk

http://dx.doi.org/10.3174/ajnr.A4792 pharyngeal wall may be undetectable by endoscopy, and they are difficult to treat. Moreover, only a small percentage of these recurrent primary tumors present early while the tumor is still amenable to salvage surgery. ${ }^{2}$ It would be beneficial to identify patients with resistant NPC so that more aggressive treatment can be given from the outset, such as an additional radiation therapy boost, chemotherapy, or targeted therapy, or these patients can be selected for posttreatment biopsy or closer posttreatment surveillance imaging.

Hypoxia and high stromal content are 2 of the factors related to a poor treatment outcome in head and neck cancers. Both micronecrosis, believed to be related to hypoxia, and high stromal content ${ }^{3}$ may decrease the restriction of the diffusion of water molecules in tumors that is reflected by an increase in the ADC on DWI. Indeed, reports of head and neck squamous cell carcinoma suggest a significant association between high pretreatment ADC and poor treatment outcome. ${ }^{4-10}$ However, for NPC, a smaller number of pretreatment predictive DWI studies have been reported. This is probably because local tumor relapse is less com- 
mon in NPC than in squamous cell carcinoma and is spread out during a longer posttreatment period. Most relapses in squamous cell carcinoma occur in the first 2 years, whereas for NPC, only around $52 \%$ of NPCs relapse in the first 2 years, with a further $39 \%$ at $2-5$ years and $9 \%$ after 5 years. ${ }^{11}$ Currently, most predictive treatment-response NPC studies are based on a relatively short-term outcome ranging up to 3 months posttreatment, ${ }^{12-14}$ and only 1 study has reported results based on longer term outcome correlating pretreatment DWI with local relapse-free or disease-free survival at 3 years. ${ }^{15}$

Therefore, the aim of this study was to determine the diagnostic performance of pretreatment DWI of the primary tumor site for the prediction of local failure (LF) based on long-term follow-up at a minimum of 5 years for patients diagnosed with local control (LC) in NPC.

\section{MATERIALS AND METHODS \\ Patients}

Patients presenting with NPC from an endemic region in southern China underwent MR imaging of the head and neck to obtain conventional anatomic-based images and DWI. Local institutional review board approval was obtained for this retrospective study. Patients were eligible for this study on the basis of the following: 1) biopsy-proved, previously untreated NPC; 2) completion of a full course of treatment with radiation therapy or chemoradiotherapy; and 3) clinical follow-up of at least 5 years from the start of treatment in patients with LC.

\section{MR Imaging Examination and Analysis}

All MR imaging examinations were performed on a $1.5 \mathrm{~T}$ whole-body system (Intera NT; Philips Healthcare, Best, the Netherlands) with a $30 \mathrm{mT} \mathrm{m}^{-1}$ maximum gradient capability. A standard receive-only head and neck coil was used. The diffusionweighted images were acquired in the axial plane by using a spinecho single-shot echo-planar imaging sequence (TR, $2000 \mathrm{~ms}$; TE, $75 \mathrm{~ms}$; section thickness, $4 \mathrm{~mm}$ without gap; FOV, $23 \mathrm{~cm}$; acquisition matrix, $112 \times 112$; reconstruction matrix, $256 \times 256$; number of signal averages, 4) with fat suppression. A pair of rectangular diffusion gradients was applied along all 3 orthogonal axes to obtain isotropic DWI with 6 b-values of $0,100,200,300,400$, and $500 \mathrm{~s} / \mathrm{mm}^{2}$. Conventional MR imaging, including axial fat-suppressed T2-weighted turbo spin-echo, axial T1-weighted spinecho, and contrast-enhanced axial T1-weighted spin-echo sequences, was also performed for anatomic correlation. DWI was performed before contrast agent injection.

\section{DWI Analysis}

The ADC map was calculated with DWI of all 6 b-values. The primary tumor in the nasopharynx was contoured on the ADC map by using the anatomic images for guidance by using the Extended MR Workspace (Philips Healthcare). Radiologic assessment was performed without knowledge of the clinical outcome. The entire volume of the primary NPC was outlined by a single radiologist (A.D.K.) with $>20$ years of experience in MR imaging of NPC. A histogram analysis method was used to examine the distribution of ADC values. The distribution of the ADC values within the primary tumor was assessed by using an in-housedeveloped Matlab (Version 7.10; MathWorks, Natick, Massachusetts) program.

The ADC parameters obtained from histogram analysis in each tumor were the ADC mean, ADC skewness, and ADC kurtosis. In this study, skewness and kurtosis are defined as $E(x-\mu)^{3} /$ $\sigma^{3}$ and $E(x-\mu)^{4} / \sigma^{4}$, respectively, where $E$ is the expected value, $\mu$ is the mean of $x$, and $\sigma$ is the SD of $x$. ADC skewness measures the skew in shape of the ADC distribution curve, with the skewness value being more positive when there is a greater frequency of low ADC values (the curve is "right-skewed" with the peak and short tail of the curve toward the left side and the long tail toward the right side) and more negative when there is a greater frequency of high ADC values (the curve is "left-skewed" with the peak and short tail of the curve toward the right side and the long tail toward the left side). ADC kurtosis measures the shape of the peak of the curve, with the kurtosis value being higher when the peak is more acute and lower when the peak is more flattened/broadened.

\section{Conventional MR Imaging Analysis}

The stage of the primary tumor (T-stage according to the seventh edition of the American Joint Committee on Cancer classification) was obtained together with primary tumor volume, calculated manually by tracing the outline of the primary nasopharyngeal tumor on the contrast-enhanced axial T1-weighted image to obtain the crosssectional area and multiplying by the section thickness.

\section{Clinical End Point Assessment}

Regular scheduled clinical follow-up was performed after treatment in all patients. LF was determined by histology (a biopsy positive for NPC at the local site at least 12 weeks after the end of treatment) or increase in tumor size on imaging or endoscopic examination. Most patients with LF present in the first 5 years; therefore, a minimum of 5-year follow-up was required in this study to confirm LC. Patients who had insufficient clinical follow-up before LC could be confirmed (including those who died within 5 years) were excluded from the study.

\section{Statistical Analysis}

The ADC parameters (mean, skewness, and kurtosis), primary tumor volume, and patient age were compared in the group of patients with LC and the group of patients with LF by using independent Student $t$ tests. Univariate logistic regression analyses with ADC parameters, primary tumor volume, patient age, and T-stage (T1-T2 versus T3-T4) were performed to determine whether there was a correlation between these parameters and LF. Odds ratios and their corresponding 95\% CI were calculated, parameters with $P$ values $<.05$ were included, and the duration of follow-up was adjusted in a multivariate analysis. Receiver operating characteristic (ROC) analysis with the area under the ROC curve was used to identify the optimal threshold of any significant parameter on multivariate analysis. The optimal threshold was obtained by optimizing the sensitivity and specificity. The sensitivity, specificity, accuracy, positive predictive value, and negative predictive value of the optimal threshold were calculated, and the significance of this threshold was re-evaluated with the $\chi^{2}$ test to ensure that it remained significant. All statistical tests were 
Table 1: ADC parameters, volume, and T-stage of the primary tumor and the patient age for prediction of treatment response

\begin{tabular}{|c|c|c|c|c|c|c|}
\hline \multirow[b]{2}{*}{ Parameter } & \multirow{2}{*}{$\begin{array}{l}\text { Whole Group } \\
\text { ( } N=158) \\
\text { (Mean) }\end{array}$} & \multirow{2}{*}{$\begin{array}{l}\text { Local Failure } \\
\quad(n=27) \\
\text { (Mean) }\end{array}$} & \multirow{2}{*}{$\begin{array}{l}\text { Local Control } \\
\qquad(n=131) \\
(\text { Mean })\end{array}$} & \multirow{2}{*}{$\begin{array}{c}\text { Student } \\
t \text { Test } \\
(P \text { Value })\end{array}$} & \multicolumn{2}{|c|}{$\begin{array}{l}\text { Univariate Logistic } \\
\text { Regression }\end{array}$} \\
\hline & & & & & $\begin{array}{c}P \\
\text { Value }\end{array}$ & $\begin{array}{c}\text { Odds Ratio } \\
(95 \% \mathrm{Cl})\end{array}$ \\
\hline $\operatorname{ADC}$ mean $\left(\times 10^{-3} \mathrm{~mm}^{2} / \mathrm{s}\right)$ & $0.91 \pm 0.11$ & $0.90 \pm 0.12$ & $0.91 \pm 0.11$ & .879 & .878 & $0.75(0.02-29.45)$ \\
\hline ADC skewness & $0.68 \pm 0.47$ & $0.45 \pm 0.40$ & $0.72 \pm 0.47$ & $.006^{\mathrm{a}}$ & $.007^{\mathrm{a}}$ & $0.22(0.08-0.67)$ \\
\hline ADC kurtosis & $4.93 \pm 1.87$ & $4.19 \pm 1.29$ & $5.08 \pm 1.94$ & $.024^{a}$ & $.024^{a}$ & $0.65(0.44-0.95)$ \\
\hline Primary tumor volume (mL) & $24.63 \pm 17.99$ & $28.20 \pm 17.90$ & $23.89 \pm 17.99$ & .256 & .298 & $1.011(0.99-1.03)$ \\
\hline \multicolumn{7}{|l|}{ T-stage } \\
\hline $\mathrm{T1}, \mathrm{T} 2$ & 74 & 12 & 62 & NA & .785 & $1.123(0.49-2.58)$ \\
\hline $\mathrm{T} 3, \mathrm{~T} 4$ & 84 & 15 & 69 & & & \\
\hline Patient age (yr) & $50.19 \pm 10.34$ & $49.74 \pm 11.34$ & $50.28 \pm 10.17$ & .805 & .804 & $1.00(0.96-1.04)$ \\
\hline
\end{tabular}

Note:-NA indicates not applicable.

a Statistically significant.

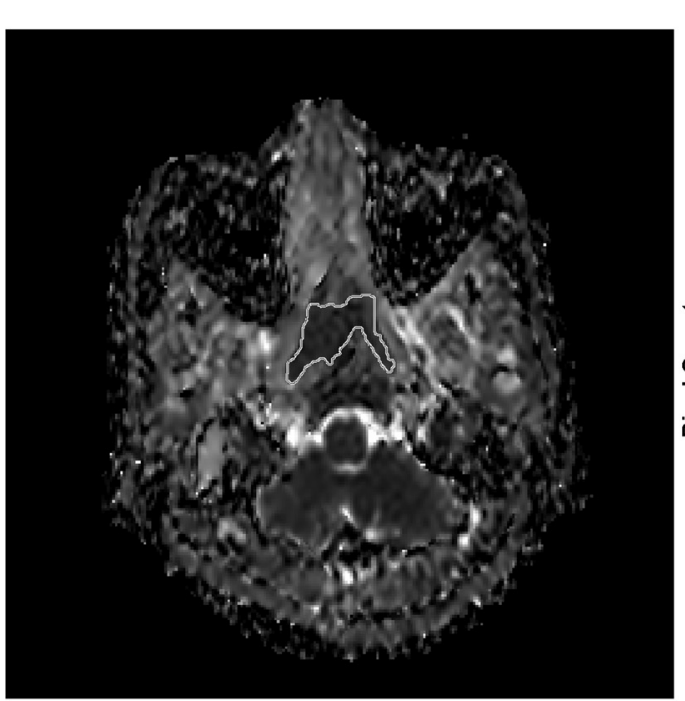

FIG 1. ADC map and histogram of primary NPC before treatment in a 50-year-old woman with local failure. The histogram shows that the greatest frequency of ADC values is shifted toward the central $A D C$ range $(A D C$ skewness $=0.11)$ and the peak is broadened $(A D C$ kurtosis $=3.89)$.

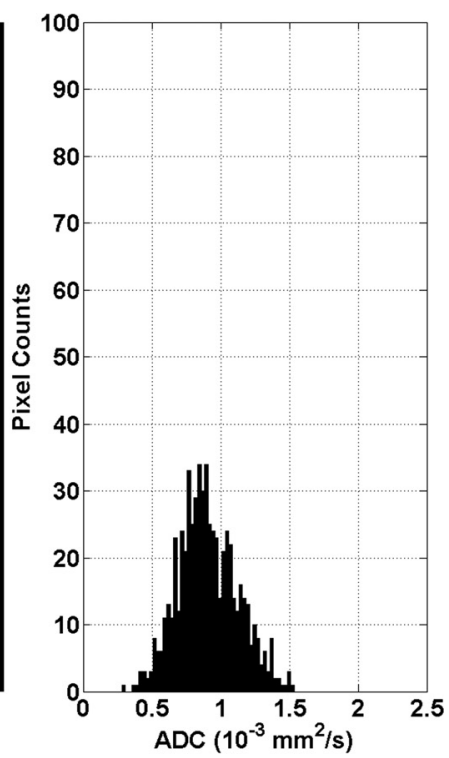

median follow-up of 87 months (mean, 88 months; range, 60.3-117.7 months) from the start of treatment. LF occurred in $27 / 158(17 \%)$ patients (undifferentiated carcinoma; $n=27$; poorly differentiated carcinoma; $n=0$ ) at a median of 25 months (mean, 33 months; range, 5.2-79.8 months) from the start of treatment. LF occurred in the first 2 years in $12 / 27$ (44\%) patients; at $2-5$ years in $11 / 27$ (41\%) patients, of whom, LF occurred in the 2- to 3 -year period in $4 / 27$ (15\%) patients; and after 5 years in $4 / 27$ (15\%) patients.

\section{DWI and Tumor Volume}

The pretreatment ADC mean, ADC skewness, ADC kurtosis, primary tumor volume, T-stage, and patient age for the group of patients with LF and the group of patients with LC and the statistical analysis are shown in Table 1. Compar2-sided, and $P$ values $<.05$ indicated a statistically significant difference. Statistical analyses were performed by using SPSS software (Version 20.0; IBM, Armonk, New York).

\section{RESULTS}

Two hundred sixty-six patients underwent DWI from March 2004 to April 2009, of whom, 108 patients were excluded from analysis for the following reasons: incomplete follow-up data $(<5$ years of clinical follow-up for patients with LC; $n=53$ ) and small lesion size/degradation of DWI for ROI analysis $(n=55)$. The study group comprised 158 patients with NPC (119 men and 39 women; mean age, 50 years; range, $27-81$ years) with undifferentiated carcinoma $(n=155)$ or poorly differentiated carcinoma $(n=3)$ who had undergone concurrent chemoradiotherapy $(n=$ $100)$ or radiation therapy alone $(n=58)$. The T-stage was T1 $(n=$ $42)$, T2 $(n=32), \mathrm{T} 3(n=56)$, and T4 $(n=28)$. The volume of the primary tumors ranged from 6.1 to $98.7 \mathrm{~mL}$, with a mean of 24.6 $\mathrm{mL}$ and a median of $18.4 \mathrm{~mL}$.

\section{Clinical End Point}

LC was achieved in 131/158 (83\%) patients (undifferentiated carcinoma; $n=128$; poorly differentiated carcinoma; $n=3$ ), with a ison of these 2 groups showed a statistically significantly lower ADC skewness (ADC values with the greatest frequencies were curve peak broader) in the group with LF (Fig 1) compared with the group with LC (Fig 2) ( $P=.006$ and .024, respectively). There was a trend toward higher tumor volumes in the group with LF, but the difference was not significant $(P=.256)$. The other parameters also showed no significant differences. ADC skewness and kurtosis significantly predicted LF in univariate analysis, but only ADC skewness remained significant $(P=.044)$ in multivariate analysis. Side-by-side boxplots of the ADC skewness values of tumors with LF and LC are shown in Fig 3. Moreover, a threshold of ADC skewness of $\leq 0.55$ ( $P=.0001)$ was identified as a predictor of LF in ROC curve analysis (Fig 4); the diagnostic performance of ADC skewness is shown in Table 2.

\section{DISCUSSION}

On the basis of long-term clinical follow-up of patients treated for NPC, pretreatment DWI showed that the ADC distribution curves of primary tumors with LF had significantly lower ADC skewness than primary tumors with LC (ie, the ADC distribution shifted away from the lower ADC range) and ADC kurtosis (ADC 

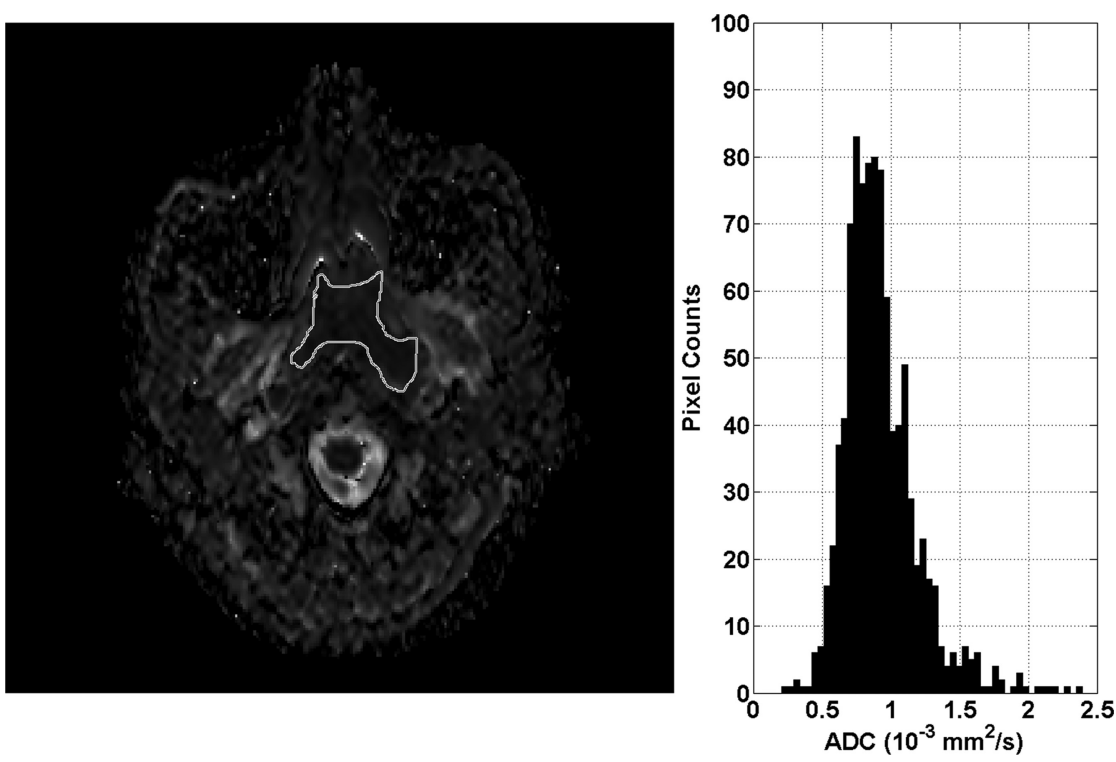

FIG 2. ADC map and histogram of primary NPC before treatment in a 43-year-old man with local control. The histogram shows that the greatest frequency of ADC values is shifted toward the lower $A D C$ range (ADC skewness $=1.42$ ) and the peak is more acute (ADC kurtosis $=8.77$ ).

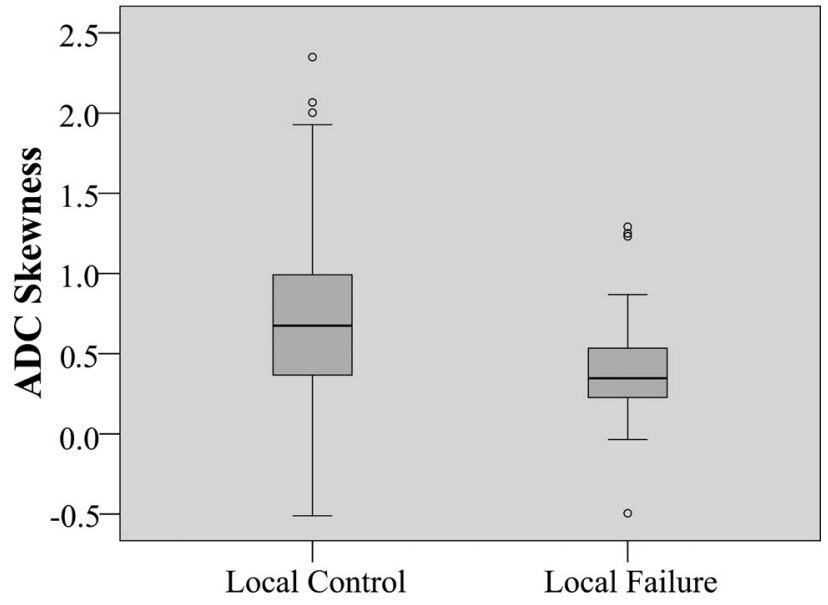

Clinical Endpoint

FIG 3. Side-by-side boxplots of ADC skewness comparing local control and local failure.

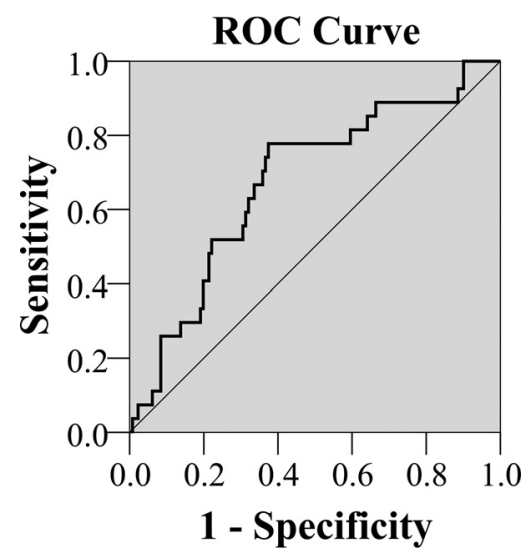

FIG 4. ROC analysis curve for ADC skewness. curve of tumors with LF showed that the greatest frequencies of $\mathrm{ADC}$ values were shifted away from the lower ADC range). Therefore, visually, compared with tumors with LC, those with LF showed a shift of the curve peak away from the left side of the curve (greater frequency of lower ADC values) toward the center (symmetric frequency distribution of low and high ADC values) or right side of the curve (greater frequency of higher ADC values). ADC skewness remained significant on multivariate analysis, and a threshold of $\leq 0.55$ produced a relatively high negative predictive value (93\%) for LF, though the positive predictive value was low (30\%), which could limit the clinical value of using DWI to predict NPC response.

Analysis of the shape of the ADC histogram curve pretreatment also showed that primary tumors with LF had significantly lower ADC kurtosis values than tumors with LC (ie, their ADC histogram peak was broader and less acute compared with the ADC histogram peak of the tumors with LC). A broader peak suggests that the tumor is more heterogeneous; this finding supports the view that those tumors with greater heterogeneity are more likely to fail treatment. ${ }^{16}$ In this study, there was also a trend toward tumors with higher volume having LF, but neither volume difference nor T-stage was significant. These results highlight the potential value of ADC skewness and ADC kurtosis because they stand out as the only parameters that may be able to identify resistant tumors over a range of tumor volumes and T-stages.

Pretreatment tumor ADC values have been shown to be predictors of treatment response in head and neck cancers. Previous head and neck squamous cell carcinoma studies have found significantly higher tumor ADC values in patients with $\mathrm{LF}^{5-7,10}$ nodal failure, ${ }^{4}$ or poor treatment response, ${ }^{8-9}$ while other studies have shown a similar trend in the ADC values that did not reach statistical significance. ${ }^{17-19}$ It has been postulated that poor outcomes of some squamous cell carcinomas are due to tumor factors that are known to increase ADC values, such as micronecrosis, lower cellularity, and, more recently, negative human papillomavirus status and high stromal content. ${ }^{3,20-21}$ Of note, the ratio of stroma to tumor cells is recognized as an important determinant of outcome in head and neck squamous cell carcinoma. In regard to NPC, research now also shows that stroma-rich NPCs are associated with poor prognosis and an increased risk of relapse. ${ }^{22}$ Therefore, it could be postulated that NPCs with high ADCs are more likely to have a poor outcome compared with those with low ADCs. Currently, there are only a few NPC DWI studies that have correlated diffusion parameters with tumor characteristics at diagnosis, such as T-stage, ${ }^{23,24}$ early intratreatment response, ${ }^{25}$ and posttreatment response. ${ }^{12-15,26}$ Regarding using pretreatment tumor ADC to predict posttreatment response, ${ }^{12-15} 3$ of these studies were based on short-term outcome at the end or 3 months after 
Table 2: ADC skewness threshold obtained from ROC curve analysis to predict local failure

\begin{tabular}{lc}
\hline \multicolumn{1}{c}{ Parameter } & ADC Skewness \\
\hline Threshold & $\leq 0.55$ \\
$P$ value & .0001 \\
Area under the ROC curve & 0.68 \\
True-positive & 21 \\
False-positive & 49 \\
True-negative & 82 \\
False-negative & 6 \\
Sensitivity & $78 \%$ \\
Specificity & $63 \%$ \\
Accuracy & $65 \%$ \\
Positive predictive value & $30 \%$ \\
Negative predictive value & $93 \%$ \\
\hline
\end{tabular}

the end of treatment. ${ }^{12-14}$ These 3 studies showed mixed results with both low ${ }^{12-13}$ and high ${ }^{14}$ pretreatment ADCs reported in tumors with poor outcomes, with the results being significant in only 1 of the studies. ${ }^{12}$ However, a recent NPC study by Zhang et $\mathrm{al}^{15}$ with long-term follow-up and a large sample size of 541 patients showed a significant association between a high mean ADC in primary tumors pretreatment and poor survival at 3 years. That study measured the pretreatment mean ADC at the level of the largest primary tumor diameter and used ROC curve analysis to identify an optimal cutoff ADC for LF of $\geq 0.747 \times 10^{-3} \mathrm{~mm}^{2} / \mathrm{s}$ (area under the ROC curve $=0.68, P=.004$ ), which was shown to correlate with both local relapse-free survival and disease-free survival.

Our study broadly supports the findings of Zhang et al, ${ }^{15}$ with high primary tumor ADC values being associated with poor local response. However, we were unable to show this correlation by using the ADC mean and could only show such a correlation by using the ADC skewness. This discrepancy between our results and those of Zhang et $\mathrm{al}^{15}$ may be related to the longer follow-up period in our study, with a subsequently greater incidence of failure at the primary site, $17 \%$ (27/158) compared with $4.3 \%$ (23/ $541)$, of which $44 \%(13 / 27)$ in our study occurred beyond 3 years.

We postulate that more sophisticated ADC measurements such as ADC skewness may be needed to identify primary tumors that will relapse at a longer time after the end of treatment. The assessment of ADC skewness in this study used histogram analysis of the distribution of the ADC values from the entire tumor volume and had an advantage over the ADC mean in that it took tumor heterogeneity into account. Most tumors are heterogeneous, and the proportion of the cancer cell population with high ADC values may influence the final treatment outcome. From the results, we postulate that tumors that are likely to have resistant tumor cells are those in which the proportion of high ADC cells is similar or greater than the proportion of low ADC cells. However, other reasons for the discrepancy in the significance of the ADC mean between the 2 studies could include the smaller sample size in our study and the difference in the outcome measures used to denote response. Specifically, we did not use survival data such as local relapse-free survival to assess primary site response; instead, we took a simple approach and directly compared the ADC values of the primary tumors with LC against the ADC values of primary tumors with LF.

Previous NPC studies have shown that larger tumor volumes are associated with more unfavorable outcomes at the primary site. ${ }^{27-35}$ This finding was also a trend in this study but did not reach significance, possibly because the mean volume of our primary tumors $(24.6 \mathrm{~mL})$ was in the lower range of previously reported cutoff thresholds, which have ranged from 13 to 60 $\mathrm{mL} .{ }^{27-35}$ In addition, the T-stage was not significant. This finding is possibly explained by the better treatment outcomes as a result of intensity-modulated radiation therapy ${ }^{36}$ and also the wide use of MR imaging for staging, which can lead to upstaging to T3 disease as a result of greater sensitivity to bone invasion compared with CT. ${ }^{37}$ Histologic NPC subtype also has a major influence on treatment outcome, whereby the undifferentiated form of NPC has a better prognosis than the other subtypes. ${ }^{38}$ In our fairly large study of 158 patients, we think that the histologic subtype did not influence the results because most (98\%) were of the same undifferentiated carcinoma subtype, with only $2 \%$ (3 tumors) being poorly differentiated, none of which showed LF.

Use of ADC measures of tumor heterogeneity such as ADC skewness and kurtosis is fairly new to the DWI research, but a few cancer studies in head and neck squamous cell carcinoma and tumors of the ovary/peritoneum and brain have shown that pretreatment ${ }^{39,40}$ or intratreatment ${ }^{19,39,40}$ ADC skewness and kurtosis may predict treatment outcome.

NPC may relapse many years after treatment; therefore, one of the main strengths of this study was the long-term clinical follow-up of the primary site (mean, $\sim 7.5$ years and maximum, $\sim 10$ years) with a minimum of 5 years for patients with LC. However, because of the long clinical follow-up required for this study, one of the main limitations was that the DWI protocol was set up some time ago when, to reduce susceptibility artifacts at the skull base, the fitted 6 b-values used were up to a maximum of 500 $\mathrm{s} / \mathrm{mm}^{2}$. It has been shown subsequently that more advanced nonGaussian models for ADC analysis influence the ADC in NPC. ${ }^{41}$ In head and neck squamous cell carcinoma, the choice of $b$-values may also influence the accuracy of ADC for the prediction of treatment response, and some authors have proposed using ADCs calculated from the mid/high b-range (300/500-1000 s/mm $\left.{ }^{2}\right)^{9,10}$ to predict locoregional response.

\section{CONCLUSIONS}

This study correlated the ADC values of the pretreatment primary NPC with treatment outcome at the primary site on the basis of long-term clinical follow-up. Compared with primary tumors with LC, those with LF had lower ADC skewness and kurtosis. The ADC skewness remained significant on multivariate analysis. The simple ADC measurement using the mean value was not a predictor of outcome in this study, suggesting that more sophisticated measurements, such as skewness, may be needed to reflect the predictive value of high ADC cancer cell populations in heterogeneous tumors. The primary tumor volume and T-stage of NPC were not significant parameters in this study for predicting treatment response at the primary site, suggesting that ADC skewness and kurtosis may have the potential to predict tumor response, even in smaller volume or earlier stage tumors.

\section{ACKNOWLEDGMENTS}

We acknowledge the assistance of H.L.E. Chan, S.T. Chan, C.H. Chen, Z. He, F.Y. Lam, and L.L. Leung. 


\section{REFERENCES}

1. Lee AW, Sze WM, Au JS, et al. Treatment results for nasopharyngeal carcinoma in the modern era: the Hong Kong experience. Int J Radiat Oncol Biol Phys 2005;61:1107-16 CrossRef Medline

2. Yu KH, Leung SF, Tung SY, et al; Hong Kong Nasopharyngeal Carcinoma Study Group. Survival outcome of patients with nasopharyngeal carcinoma with first local failure: a study by the Hong Kong Nasopharyngeal Carcinoma Study Group. Head Neck 2005;27:397405 CrossRef Medline

3. Driessen JP, Caldas-Magalhaes J, Janssen LM, et al. Diffusionweighted MR imaging in laryngeal and hypopharyngeal carcinoma: association between apparent diffusion coefficient and histologic findings. Radiology 2014;272:456-63 CrossRef Medline

4. Kim S, Loevner L, Quon H, et al. Diffusion-weighted magnetic resonance imaging for predicting and detecting early response to chemoradiation therapy of squamous cell carcinomas of the head and neck. Clin Cancer Res 2009;15:986-94 CrossRef Medline

5. Hatakenaka M, Nakamura K, Yabuuchi H, et al. Pretreatment apparent diffusion coefficient of the primary lesion correlates with local failure in head-and-neck cancer treated with chemoradiotherapy or radiotherapy. Int J Radiat Oncol Biol Phys 2011;81:339-45 CrossRef Medline

6. Hatakenaka M, Shioyama Y, Nakamura K, et al. Apparent diffusion coefficient calculated with relatively high b-values correlates with local failure of head and neck squamous cell carcinoma treated with radiotherapy. AJNR Am J Neuroradiol 2011;32:1904-10 CrossRef Medline

7. Ohnishi K, Shioyama Y, Hatakenaka M, et al. Prediction of local failures with a combination of pretreatment tumor volume and apparent diffusion coefficient in patients treated with definitive radiotherapy for hypopharyngeal or oropharyngeal squamous cell carcinoma. J Radiat Res 2011;52:522-30 CrossRef Medline

8. Srinivasan A, Chenevert TL, Dwamena BA, et al. Utility of pretreatment mean apparent diffusion coefficient and apparent diffusion coefficient histograms in prediction of outcome to chemoradiation in head and neck squamous cell carcinoma. J Comput Assist Tomogr 2012;36:131-37 CrossRef Medline

9. Lambrecht $M$, Van Calster B, Vandecaveye V, et al. Integrating pretreatment diffusion weighted MRI into a multivariable prognostic model for head and neck squamous cell carcinoma. Radiother Oncol 2014;110:429-34 CrossRef Medline

10. Hatakenaka M, Nakamura K, Yabuuchi H, et al. Apparent diffusion coefficient is a prognostic factor of head and neck squamous cell carcinoma treated with radiotherapy. Jpn J Radiol 2014;32:80-89 CrossRef Medline

11. Lee AW, Foo W, Law SC, et al. Recurrent nasopharyngeal carcinoma: the puzzles of long latency. Int J Radiat Oncol Biol Phys 1999;44:149-56 CrossRef Medline

12. Zheng D, Chen Y, Chen Y, et al. Early assessment of induction chemotherapy response of nasopharyngeal carcinoma by pretreatment diffusion-weighted magnetic resonance imaging. J Comput Assist Tomogr 2013;37:673-80 CrossRef Medline

13. Chen Y, Liu X, Zheng D, et al. Diffusion-weighted magnetic resonance imaging for early response assessment of chemoradiotherapy in patients with nasopharyngeal carcinoma. Magn Reson Imaging 2014;32:630-37 CrossRef Medline

14. Hong J, Yao Y, Zhang Y, et al. Value of magnetic resonance diffusion-weighted imaging for the prediction of radiosensitivity in nasopharyngeal carcinoma. Otolaryngol Head Neck Surg 2013;149: 707-13 CrossRef Medline

15. Zhang Y, Liu X, Zhang Y, et al. Prognostic value of the primary lesion apparent diffusion coefficient (ADC) in nasopharyngeal carcinoma: a retrospective study of $\mathbf{5 4 1}$ cases. Sci Rep 2015;5:12242 CrossRef Medline

16. Mroz EA, Tward AD, Pickering CR, et al. High intratumor genetic heterogeneity is related to worse outcome in patients with head and neck squamous cell carcinoma. Cancer 2013;119:3034-42 CrossRef Medline
17. Matoba M, Tuji H, Shimode Y, et al. Fractional change in apparent diffusion coefficient as an imaging biomarker for predicting treatment response in head and neck cancer treated with chemoradiotherapy. AJNR Am J Neuroradiol 2014;35:379-85 CrossRef Medline

18. Chawla S, Kim S, Dougherty L, et al. Pretreatment diffusionweighted and dynamic contrast-enhanced MRI for prediction of local treatment response in squamous cell carcinomas of the head and neck. AJR Am J Roentgenol 2013;200:35-43 CrossRef Medline

19. King $\mathrm{AD}$, Chow $\mathrm{KK}, \mathrm{Yu} \mathrm{KH}$, et al. Head and neck squamous cell carcinoma: diagnostic performance of diffusion-weighted MR imaging for the prediction of treatment response. Radiology 2013;266: 531-38 CrossRef Medline

20. Driessen JP, van Bemmel AJ, van Kempen PM, et al. Correlation of human papillomavirus status with apparent diffusion coefficient of diffusion-weighted MRI in head and neck squamous cell carcinomas. Head Neck 2015 Mar 17. [Epub ahead of print] CrossRef Medline

21. Nakahira M, Saito N, Yamaguchi H, et al. Use of quantitative diffusion-weighted magnetic resonance imaging to predict human papilloma virus status in patients with oropharyngeal squamous cell carcinoma. Eur Arch Otorhinolaryngol 2014;271:1219-25 CrossRef Medline

22. Zhang XL, Jiang C, Zhang ZX, et al. The tumor-stroma ratio is an independent predictor for survival in nasopharyngeal cancer. $\mathrm{On}$ col Res Treat 2014;37:480-84 CrossRef Medline

23. Abdel Razek AA, Kamal E. Nasopharyngeal carcinoma: correlation of apparent diffusion coefficient value with prognostic parameters. Radiol Med 2013;118:534-39 CrossRef Medline

24. Lai V, Li X, Lee VH, et al. Nasopharyngeal carcinoma: comparison of diffusion and perfusion characteristics between different tumour stages using intravoxel incoherent motion MR imaging. Eur Radiol 2014;24:176-83 CrossRef Medline

25. Chen Y, Ren W, Zheng D, et al. Diffusion kurtosis imaging predicts neoadjuvant chemotherapy responses within 4 days in advanced nasopharyngeal carcinoma patients. J Magn Reson Imaging 2015;42: 1354-61 CrossRef Medline

26. Xu JF, Wu XW, Wang WQ, et al. Value of diffusion-weighted magnetic resonance imaging on the follow-up of nasopharyngeal carcinoma after radiotherapy. J Xray Sci Technol 2014;22:605-12 CrossRef Medline

27. Wu Z, Su Y, Zeng RF, et al. Prognostic value of tumor volume for patients with nasopharyngeal carcinoma treated with concurrent chemotherapy and intensity-modulated radiotherapy. J Cancer Res Clin Oncol 2014;140:69-76 CrossRef Medline

28. Feng M, Wang W, Fan Z, et al. Tumor volume is an independent prognostic indicator of local control in nasopharyngeal carcinoma patients treated with intensity-modulated radiotherapy. Radiat Oncol 2013;8:208 CrossRef Medline

29. Guo R, Sun Y, Yu XL, et al. Is primary tumor volume still a prognostic factor in intensity modulated radiation therapy for nasopharyngeal carcinoma? Radiother Oncol 2012;104:294-99 CrossRef Medline

30. Lee CC, Huang TT, Lee MS, et al. Clinical application of tumor volume in advanced nasopharyngeal carcinoma to predict outcome. Radiat Oncol 2010;5:20 CrossRef Medline

31. Shen C, Lu JJ, Gu Y, et al. Prognostic impact of primary tumor volume in patients with nasopharyngeal carcinoma treated by definitive radiation therapy. Laryngoscope 2008;118:1206-10 CrossRef Medline

32. Kim JH, Lee JK. Prognostic value of tumor volume in nasopharyngeal carcinoma. Yonsei Med J 2005;46:221-27 CrossRef Medline

33. Sze WM, Lee AW, Yau TK, et al. Primary tumor volume of nasopharyngeal carcinoma: prognostic significance for local control. Int J Radiat Oncol Biol Phys 2004;59:21-27 CrossRef Medline

34. Chua DT, Sham JS, Leung LH, et al. Tumor volume is not an independent prognostic factor in early-stage nasopharyngeal carcinoma treated by radiotherapy alone. Int J Radiat Oncol Biol Phys 2004;58:1437-44 CrossRef Medline

35. Chua DT, Sham JS, Kwong DL, et al. Volumetric analysis of tumor 
extent in nasopharyngeal carcinoma and correlation with treatment outcome. Int J Radiat Oncol Biol Phys 1997;39:711-19 CrossRef Medline

36. Lai SZ, Li WF, Chen L, et al. How does intensity-modulated radiotherapy versus conventional two-dimensional radiotherapy influence the treatment results in nasopharyngeal carcinoma patients? Int J Radiat Oncol Biol Phys 2011;80:661-68 CrossRef Medline

37. Liao XB, Mao YP, Liu LZ, et al. How does magnetic resonance imaging influence staging according to AJCC staging system for nasopharyngeal carcinoma compared with computed tomography? Int J Radiat Oncol Biol Phys 2008;72:1368-77 CrossRef Medline

38. Cheng SH, Tsai SY, Horng CF, et al. A prognostic scoring system for locoregional control in nasopharyngeal carcinoma following con- formal radiotherapy. Int J Radiat Oncol Biol Phys 2006;66:992-1003 CrossRef Medline

39. Kyriazi S, Collins DJ, Messiou C, et al. Metastatic ovarian and primary peritoneal cancer: assessing chemotherapy response with diffusion-weighted MR imaging-value of histogram analysis of apparent diffusion coefficients. Radiology 2011;261:182-92 CrossRef Medline

40. Nowosielski M, Recheis W, Goebel G, et al. ADC histograms predict response to anti-angiogenic therapy in patients with recurrent high-grade glioma. Neuroradiology 2011;53:291-302 CrossRef Medline

41. Yuan J, Yeung DK, Mok GS, et al. Non-Gaussian analysis of diffusion weighted imaging in head and neck at $3 \mathrm{~T}$ : a pilot study in patients with nasopharyngeal carcinoma. PLoS One 2014;9:e87024 CrossRef Medline 